\title{
Influence of Periapical Lesion on Healing in Sinus after Endodontics
}

\author{
Klaudia Migas ${ }^{1}$ Joanna Marchlewska ${ }^{2}$ \\ ${ }^{1}$ Department of Health Science, Medical University of Lodz, Lodz, Poland \\ 2 Department of Head and Neck Surgery, Joanna Marchlewska, The \\ Greater Poland Cancer Centre, University of Medical Sciences \\ Poznan, Poznan, Poland \\ Eur J Gen Dent 2022;11:46-50.
}

Address for correspondence Klaudia Migas, DDS, MSc, Department of Health Science, Medical University of Lodz, 90-001 Lodz, Plac Hallera 1, Poland (e-mail: migas.klaudia@gmail.com).

\author{
Abstract \\ Keywords \\ - root canal treatment \\ - Schneider's \\ membrane \\ - maxillary sinus \\ inflammation \\ - periapical lesion
}

Objective The goal of primary and secondary endodontic treatment is to eliminate the cause of inflammation inside the tooth and in the surrounding tissues. When the inflammation from the root canal system of the tooth spreads beyond the apex of the root, periapical changes in the bone tissue and, in the case of upper premolars and molars, inflammatory changes in the Schneider membrane may occur.

Materials and Methods In a retrospective documentation analysis of root canal treatments, three-dimensional (3D) computed tomography images before and after endodontic treatment were assessed to measure the thickness of the Schneider membrane. Forty-five endodontically treated patients aged 21 to 62 years were enrolled in the study. Inflammation of the maxillary sinus was considered when the Schneider membrane was thicker than $2 \mathrm{~mm}$. Statistical analyses were performed with Statistica 12 by StatSoft and StatXact by Cytel using the Shapiro-Wilk test, Student's ttest, and the Mann-Whitney test.

Results Out of 12 comparisons between variables, only 3 comparisons were significant. There was a relationship between the treatment effect (reduction in maxillary sinus inflammation 3 months after treatment) and the maximum height of the Schneider membrane $(p=0.004)$. There was a relationship between the presence of a periapical lesion and the minimum height of the Schneider diaphragm $(p=0.02)$, and there was a relationship between the presence of a periapical lesion and the maximum height of the Schneider diaphragm $(p=0.04)$.

Conclusion Primary and secondary root canal treatments of maxillary premolars and molars reduce the inflammation of the maxillary sinus 3 months after treatment.

\section{Introduction}

Contemporary antiseptic root canal treatment (or endodontic treatment) consists of removing the infected pulp and root canal dentine from the tooth cavity through biomechanical and chemical preparation of the root canal system and then filling them securely.
The indications for antiseptic root canal treatment include irreversible pulpopathy with viable pulp with purulent exudate and necrotic foci, with dead pulp, or with gangrenous decay; apical periodontitis related to pulpitis or gangrene; and root canal retreatment.

The prognosis for antiseptic root canal treatment is good, provided that the treatment is performed in accordance with

\section{(C) 2022. The Author(s).}

This is an open access article published by Thieme under the terms of the Creative Commons Attribution License, permitting unrestricted use, distribution, and reproduction so long as the original work is properly cited. (https://creativecommons.org/licenses/by/4.0/)

Thieme Medical and Scientific Publishers Pvt. Ltd., A-12, 2nd Floor, Sector 2, Noida-201301 UP, India 
the applicable standards (chemomechanical removal of the infected tissue and complete filling).

Improvement or elimination of apical lesions within a few months after the end of treatment indicates a good result of endodontic treatment. For this reason, periodic clinical and radiological check-ups are recommended after antiseptic root canal treatment. ${ }^{1}$

As a result of a lack of treatment of the inflammation of the tooth pulp, bacteria and toxins can penetrate beyond the tooth tip, causing inflammation of the surrounding tissues, including bone, soft tissue, and the maxillary sinus. ${ }^{2-5}$

The roots of the upper premolars and upper molars are located near the bottom of the maxillary sinus; hence, the spread of inflammation into the sinus lumen can occur. ${ }^{5}$

Changes in the maxillary sinus may be asymptomatic or symptomatic; in the case of an asymptomatic course, no treatment is recommended. These changes are often diagnosed accidentally during a three-dimensional (3D) radiological examination during a dental visit. ${ }^{2,4}$

Odontogenic changes in the maxillary sinus are mitigated by treating the cause, i.e., endodontic treatment or removal of the tooth, among others. Tooth extraction ensures immediate removal of the cause of the infection. ${ }^{1}$ However, most patients want to leave the tooth in the mouth, so when the cause of infection is irreversible inflammation of the tooth pulp, the treatment of choice is root canal treatment. The aim of the study was to check whether properly performed endodontic treatment ensures the healing process of the maxillary sinus inflammation and how long the healing process takes place in the maxillary sinuses. In the case of periapical changes, the first control X-ray is taken 6 months after the end of endodontic treatment. In the case of inflammation of the maxillary sinuses, the healing test was checked 3 months after the end of treatment on $\mathrm{CBCT}^{6}$ Assessing whether healing of maxillary sinus inflammation is visible on X-rays earlier than 6 months is clinically relevant to the clinical management of the patient. ${ }^{4,5}$ The possibility of earlier evaluation of the healing process of the maxillary sinuses postpones the decision on surgical and laryngologist treatment of maxillary sinus inflammation, and thus reduces the frequency of making decisions about surgical treatment. In the case of the standard waiting period for an X-ray examination, 6 months after the end of endodontic treatment, surgical treatment was associated with chronic sinusitis. Surgical treatment of sinusitis is associated with irreversible ingeneration into the Schneider membrane, its partial removal, adhesions, alveolar bone loss, and more difficult conditions for future dental implantation. ${ }^{3}$

In the pathogenesis of odontogenic maxillary sinusitis, bacteria that occur in the oral cavity, especially those causing pulpitis, play a major role. Bacterial flora affects the form of acute maxillary sinusitis: serous or purulent. In chronic sinusitis, in addition to the purulent form, there is a proliferative form. In acute inflammations, hyperemia and swelling of the mucosa of the maxillary sinus occur, and discharge is observed. In chronic inflammations, the type of changes that occur depends not only on the type of bacterial flora but also on the duration of the process, its intensity, and exacerbations of the inflammatory process. In these conditions, hyperplastic hypertrophy and polyps, which may be accompanied by serous-purulent discharge, are observed. In other cases, the Schneider diaphragm is lost, thinned, and overgrown. These conditions are accompanied by purulent discharge. ${ }^{2-5}$

In the consolidation of the chronic state of the maxillary sinuses, foci of infection in the vicinity play a significant role, in addition to the biological resistance of the mucosa, obstruction of natural orifices, impaired ability to absorb exudate or acute recurrences. ${ }^{4}$

In cases of outbreaks of infection in the vicinity, it is often possible to establish the cause of the maxillary premolar or molar. The 3D conical computed tomography diagnosis facilitates diagnosis. ${ }^{2,5}$

Inflammation of the maxillary sinuses is accompanied by both local and general symptoms, which result mainly from drainage and ventilation disorders caused by infection.

Patients coming to the dentist report symptoms characteristic of the inflammation of the maxillary sinuses should have a radiological diagnosis of the maxillary sinuses, if Schneider's membrane hypertrophy is confirmed, such condition can be successfully treated by a dentist endodontically and then checked on X-rays and depending on the result of the healing process referred or not to a laryngologist specialist. ${ }^{4}$

During the interview and examination, patients report pain in the projection of the affected maxillary sinus, a feeling of tension, malaise, difficulty breathing through the nose, fever, discharge of discharge through the nose, and palpation pain on the front wall of the maxillary sinus. In chronic inflammation, these symptoms may or may not be more discrete. ${ }^{3-5}$

The diagnosis is made on the basis of a physical and physical examination, and the diagnosis is confirmed by radiological diagnosis, which shows the degree of sinus shading, the presence of fluid, or hypertrophy of the mucosa of the maxillary sinus (Schneider's membrane).

The treatment of acute maxillary sinusitis begins with the elimination of the cause, i.e., the removal of the causative tooth or its root canal treatment, along with the observation of the healing process. Chronic conditions require the implementation of laryngologist surgery. This requires access to the maxillary sinus through the alveolar bone or the anterior nostrils, depending on the treatment technique. Surgical treatment consists of gaining access to the maxillary sinus, removing its contents together with a fragment of macroscopically changed mucosa, and enlarging the nasal outflow opening in the medial wall. ${ }^{1}$

Treatment of odontogenic maxillary sinusitis requires close cooperation between a dentist and a laryngologist specialist to ensure the optimal course of treatment. ${ }^{2,3}$

\section{Thesis Objective}

The aim of the study was to check whether inflammation of the maxillary sinus decreased after endodontic treatment of the upper premolars and molars and whether variables such as sex, patient age, the presence of a periapical lesion, and the type of primary or secondary root canal treatment affected the healing process. 


\section{Research Questions}

1. Does endodontic treatment of the upper premolars and upper molars reduce inflammation of the maxillary sinus?

2. Do the sex and age of the treated person affect the inflammation healing process in the maxillary sinus?

3. Does the presence of a periapical lesion affect the inflammation healing process in the maxillary sinus?

4. Is the inflammation healing process in the maxillary sinus dependent on whether endodontic treatment is primary or secondary?

\section{Materials and Methods}

Forty-five people whose teeth were endodontically treated at a dental clinic in Krakow, Poland, from January 2019 to June 2020, were enrolled in this retrospective study.

The study included people whose upper premolars or molars required primary or secondary endodontic treatment, who had undergone a 3D computed tomography examination with a Carestream 9300 Premium device, who had received root canal treatment before or within 3 months after treatment, and who had received root canal treatment with an epoxy resin sealant.

People who had a history of symptoms in the paranasal sinuses and had inflammation of both maxillary sinuses were excluded from the study. Patients who were enrolled in the study, during endodontic treatment and 3 months after the end of treatment, were not treated with any drugs, including anti-inflammatory drugs.

Forty-five people aged 21 to 62 years were enrolled in the study; $58 \%$ were men, and $42 \%$ were women. In total, $53 \%$ of teeth that qualified for endodontic treatment showed the presence of a periapical lesion before treatment, and in 69\% of cases, inflammation of the maxillary sinus was present before treatment. The proportions of analyzed premolars and molars were $42 \%$ and $58 \%$, respectively.

The presence of periapical lesions in maxillary premolars and molars was determined by the CBCT periapical index images during root canal treatment and 3 months after treatment.

The measurements were made in the projection of the tops of the root canal-treated tooth at two measurement points from the lowest to the highest measurement point. The bottom of the maxillary sinus in the projection of the tops of the root canal-treated tooth was taken as the lowest measurement point, and the highest point of erosion of the Schneider membrane hypertrophy was taken as the highest measurement point. The distance was measured in millimeters $(\mathrm{mm})$. Distance measurements were made with Carestream computed tomography software in orthopantomographic projections with a resolution of 75 microns.

The condition of the periapical tissues and the presence of periapical changes were assessed on the basis of the CBCT periapical index (CBCTPAI score). The CBCTPAI score is assessed for the largest lesion size, measured in the buccopalatal, mesiodistal, and diagonal dimensions. In multi-root- ed teeth, the index was assessed at the root with the greatest periapical change.

All measurements were made according to a standardized method. Each of the CBCT images was reviewed by two dentists: one general dentist and one endodontist. Measurements for each CBCT image were taken twice at monthly intervals.

In cases where the measurement difference was $>0.2 \mathrm{~mm}$ between observers, the measurement was re-taken and the average of the measurement was taken into account.

The maximum size of periapical changes was taken into account in the three planes: axial, coronal, and sagittal, and included in millimeters.

For the maxillary sinus, the maximum hypertrophy of the Schneiderian membrane was $31.7 \mathrm{~mm}$. Maxillary premolars and molars with lesions up to $5 \mathrm{~mm}$ and $5 \mathrm{~mm}$ were qualified for the study. The largest periapical change of the tooth in the study was $12 \mathrm{~mm}$.

In this retrospective study of root canal treatments, the diagnosis was performed before and after endodontic treatment by measuring the thickness of the Schneider membrane on 3D computed tomography. Inflammation of the maxillary sinus was considered when the Schneider membrane was more than $2 \mathrm{~mm}$ thick.

The results were calculated on the basis of the following variables: age, sex, tooth number, presence of a periapical lesion, and type of root canal treatment. Statistical analyses were performed using Statistica 12 by StatSoft and StatXact by Cytel, using the Shapiro-Wilk test, Student's $t$-test, and the Mann-Whitney test.

\section{Results}

The calculations were made using Statistica 12 by StatSoft and StaatXact by Cytel. $\propto=0.05$ was adopted as the level of significance. The result was considered statistically significant when $p$ was less than $\propto$. The normality of the distribution of variables was checked using the Shapiro-Wilk test. A test was used to compare the variables between the two groups: $t$-Student's for unrelated samples, in case of compliance with the normal distribution and equal variances, or the Mann-Whitney test.

To test the relationship between categorical variables, the Chi-square test of independence, the Fischer exact test, or the Fischer-Freeman-Halton test were calculated.

The data distribution was normal, at $p>0.05$. There was a relationship between the presence of a periapical lesion and the minimum (-Fig. 1) and maximum ( - Fig. 2) height of the Schneider membrane before endodontic treatment. There was a relationship between the decrease in inflammation in the maxillary sinus and the maximum height of the Schneider membrane (-Fig. 3). Correctly performed endodontic treatment allows for a reduction in inflammation in the maxillary sinus to be observed as early as 3 months after the end of treatment. There was a relationship between endodontic treatment and a reduction in maxillary sinus inflammation. 


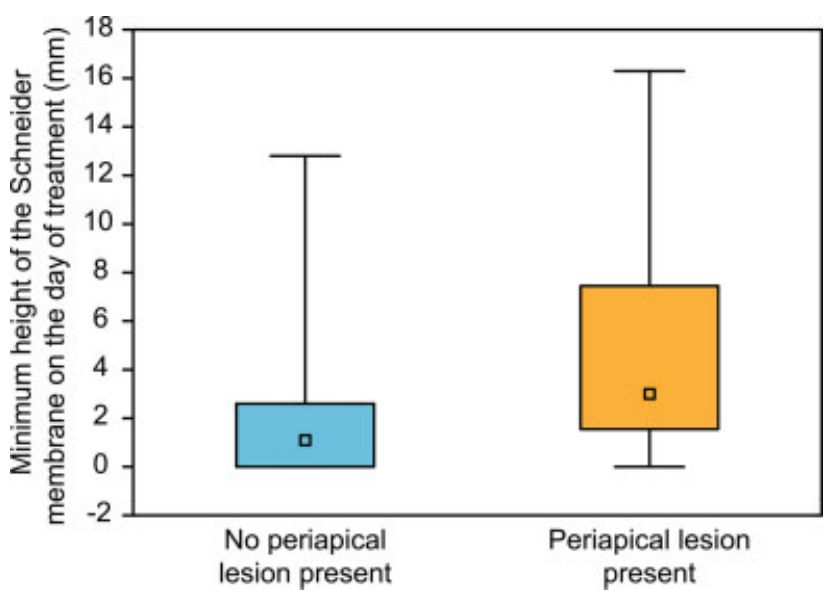

Fig. 1 Minimum height of the Schneider membrane on the day of treatment $(\mathrm{mm})$.

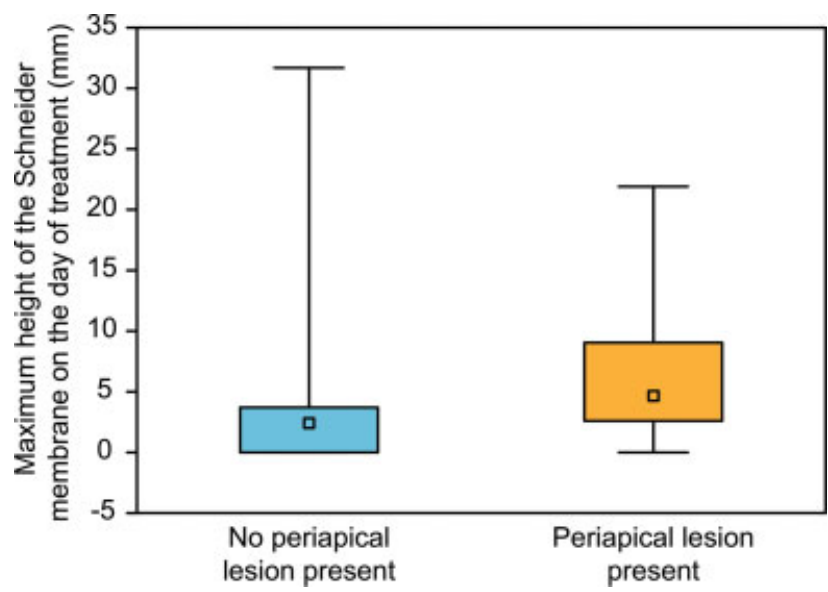

Fig. 2 Maximum height of the Schneider membrane on the day of treatment $(\mathrm{mm})$.

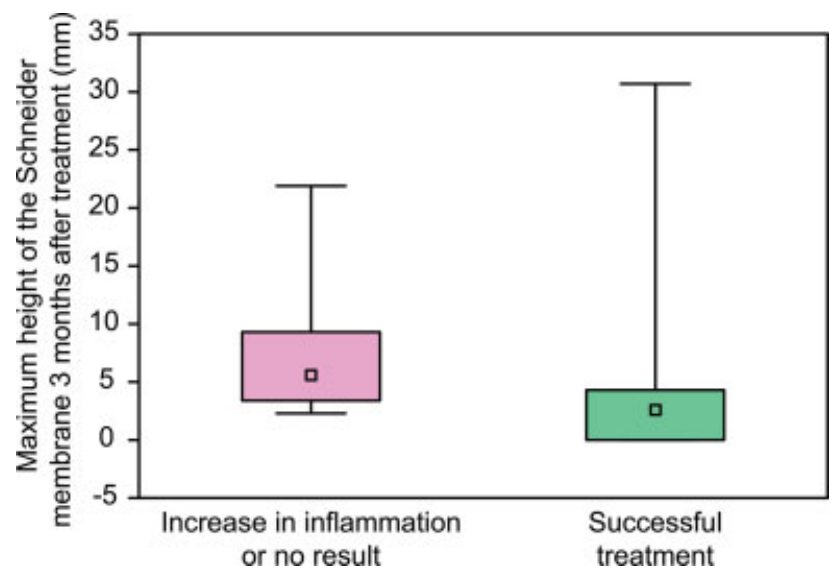

Fig. 3 Maximum height of the Schneider membrane 3 months after treatment $(\mathrm{mm})$.

There were no effects of age, sex, periapical lesion, type of tooth, or type of endodontic treatment (primary, secondary) on the inflammation healing process in the maxillary sinus after 3 months.
The presence of a periapical lesion did not affect the inflammation healing process in the maxillary sinus after endodontic treatment of a tooth.

\section{Discussion}

Correctly performed endodontic treatment allows the removal of the cause of inflammation in the causative tooth and from the surrounding tissues, e.g., bones, gums, and the maxillary sinus. ${ }^{7,8}$

Secondary endodontic treatment has been associated with less success than primary treatment; secondary treatment could potentially influence the healing process in the surrounding tissues, i.e., the maxillary sinus. ${ }^{9}$ Additionally, an unfavorable healing process could be influenced by different bacterial flora in the root systems of teeth previously endodontically treated. ${ }^{10,11}$

A retrospective analysis of treatment at a dental clinic in Krakow did not confirm this. Whether endodontic treatment was primary or secondary had no impact on the process in the maxillary sinus. Appropriate root canal treatment of the tooth is significant. ${ }^{12}$

Patient-related factors, such as sex and age, had no influence on the process in the maxillary sinus. The age of the person undergoing treatment may have an influence on the healing process, as healing ability potentially declines with age; however, this study found no influence of age or sex on the process in the maxillary sinus. ${ }^{13} 14,15$

It should be stated that the presence of a periapical lesion did not affect the healing process in the maxillary sinus.

It did not matter whether the treated tooth was a premolar or a molar; the most important factor influencing the healing process was appropriate endodontic treatment. ${ }^{16,17}$

After 3 months, a reduction in sinus inflammation was evident, and computed tomography scans of the affected area are warranted to assess the healing process of odontogenic sinus inflammation. ${ }^{18-20}$

\section{Conclusions}

Correctly performed endodontic treatment reduced inflammation in the maxillary sinus on the same side as the tooth that received root canal treatment. Decreasing odontogenic inflammation of the maxillary sinus on the same side as the endodontically treated tooth can be diagnosed as early as 3 months after the treatment.

The results of the study show that it is possible to effectively treat the inflammation of the maxillary sinuses through conservative treatment, i.e., endodontic treatment of the tooth. These results allow changing the current recommendations for the treatment of maxillary sinus inflammation, which were based on irreversible removal of the causal tooth from the oral cavity and surgical (surgical) treatment of the maxillary sinus inflammation.

The results of the study indicate that it is possible to reduce the inflammation of the maxillary sinuses of dental origin after endodontic treatment of the causative tooth, and thus allow the causal tooth to be left from the oral cavity and 
reduce the need for surgical treatment of the inflammation of the maxillary sinuses of dental origin.

It is important that the reduction of the inflammation of the maxillary sinuses of dental origin takes place regardless of the type of treatment, i.e., primary endodontic treatment or secondary endodontic treatment. The gender or age of the treated person did not affect the success of treatment either.

An important result is a fact that the periapical lesion has no effect on the healing process in the maxillary sinus, and the fact that the healing process can already be diagnosed on X-ray examinations 3 months after the end of endodontic treatment, and not, as in the case of observation of the healing of periapical lesions, only after 6 months.

Shortening the waiting time for the test result and observation of the healing process allows the dentist to make a faster decision about the need for a laryngologist consultation in the absence of the healing process.

The results of the study indicate the need for cooperation between a dentist and a laryngologist in the treatment of maxillary sinus inflammation.

\section{Conflict of Interest}

None declared.

\section{References}

1 Starkey JL, Mortman RE. Treatment of maxillary sinusitis of odontogenic origin: a case series. Compend Contin Educ Dent 2019;40(08):516-522

2 Grygorov S, Poberezhnik G, Grygorova A. Actual issues of odontogenic maxillary sinusitis (review). Georgian Med News 2018; (276):46-50

3 Taschieri S, Torretta S, Corbella S, et al. Pathophysiology of sinusitis of odontogenic origin. J Investig Clin Dent 2017;8(02). Doi: $10.1111 /$ jicd. 12202

4 Mehra P, Jeong D. Maxillary sinusitis of odontogenic origin. Curr Allergy Asthma Rep 2009;9(03):238-243

5 de Lima CO, Devito KL, Baraky Vasconcelos LR, Prado MD, Campos $\mathrm{CN}$. Correlation between endodontic infection and periodontal disease and their association with chronic sinusitis: a clinicaltomographic study. J Endod 2017;43(12):1978-1983

6 Tabassum S, Khan FR. Failure of endodontic treatment: the usual suspects. Eur J Dent 2016;10(01):144-147

7 Del Fabbro M, Corbella S, Sequeira-Byron P, et al. Endodontic procedures for retreatment of periapical lesions. Cochrane Database Syst Rev 2016;10:CD005511. Doi:10.1002/14651858.CD005511.pub3
8 Wei MY, Wang XL, Li Y, Gao Y. Study on root canal variation of maxillary premolar and its relationship to maxillary sinus by cone-beam CT [article in Chinese]. Shanghai Kou Qiang Yi Xue 2018;27(02):156-163. Last accessed on January 18, 2022, at: sjos.cn/EN/abstract/abstract6801.shtml

9 Ng YL, Mann V, Gulabivala K. Outcome of secondary root canal treatment: a systematic review of the literature. Int Endod J 2008; 41(12):1026-1046. Doi:10.1111/j.1365-2591.2008.01484.x

10 Pereira RS, Rodrigues VAA, Furtado WT, Gueiros S, Pereira GS, Avila-Campos MJ. Microbial analysis of root canal and periradicular lesion associated to teeth with endodontic failure. Anaerobe 2017;48:12-18. Doi:10.1016/j.anaerobe.2017.06.016

11 Meirinhos J, Martins JNR, Pereira B, et al. Prevalence of apical periodontitis and its association with previous root canal treatment, root canal filling length and type of coronal restoration - a cross-sectional study. Int Endod J 2020;53(04):573-584. Doi: $10.1111 /$ iej.13256

12 Lin LM, Ricucci D, Lin J, Rosenberg PA. Nonsurgical root canal therapy of large cyst-like inflammatory periapical lesions and inflammatory apical cysts. J Endod 2009;35(05):607-615. Doi: 10.1016/j.joen.2009.02.012

13 Jahreis M, Soliman S, Schubert A, et al. Outcome of non-surgical root canal treatment related to periodontitis and chronic disease medication among adults in age group of 60 years or more. Gerodontology 2019;36(03):267-275. Doi:10.1111/ger.12407

14 Mustafa NS, Kashmoola MA, Majeed KRA, Qader OAJA. Assessment of the success rate of endodontically treated patients attending outpatient polyclinic. Eur J Dent 2018;12(04):540-545

15 Gambarini G, Piasecki L, Miccoli G, Gaimari G, Nardo DD, Testarelli L. Cone-beam computed tomography in the assessment of periapical lesions in endodontically treated teeth. Eur J Dent 2018;12 (01):136-143

16 Yousuf W, Khan M, Sheikh A. Success rate of overfilled root canal treatment. J Ayub Med Coll Abbottabad 2015;27(04):780-783

17 Iikubo M, Sasano T, Shoji N, Sakamoto M. Nonsurgical treatment for odontogenic maxillary sinusitis using irrigation through the root canal: preliminary case report. Tohoku J Exp Med 2002;197 (01):47-53. Doi:10.1620/tjem.197.47

18 Restrepo-Restrepo FA, Cañas-Jiménez SJ, Romero-Albarracín RD, Villa-Machado PA, Pérez-Cano MI, Tobón-Arroyave SI. Prognosis of root canal treatment in teeth with preoperative apical periodontitis: a study with cone-beam computed tomography and digital periapical radiography. Int Endod J 2019;52(11):1533-1546. Doi: 10.1111/iej.13168

19 Nair PN. On the causes of persistent apical periodontitis: a review. Int Endod J 2006;39(04):249-281. Doi:10.1111/j.1365-2591.2006. 01099.x

20 Starkey JL, Mortman RE. Treatment of maxillary sinusitis of odontogenic origin: a case series. Compend Contin Educ Dent 2019;40(08):516-522 